\title{
Circumferential Pupillary Capture by IOL due to Intraocular Gas Tamponade: A Case Report
}

\author{
Mesut Erdurmuş*, Uğur Acar and Güngör Sobacı \\ Department of Ophthalmology, Hacettepe University Medical School, Ankara, Turkey
}

*Corresponding author: Mesut Erdurmus, MD, Department of Ophthalmology, Hacettepe University Medical School, 06100 Sihhiye, Ankara, Turkey, Tel: +90-505-525-1135, Fax: +90-312-309-4101

\begin{abstract}
A 55-years-old female presented with pain in the right eye for 15 days. She had a history of complicated cataract surgery followed by pars plana vitrectomy, intraocular gas injection and sulcus fixated intraocular lens implantation. On ophthalmologic examination, pupillary capture by intraocular lens, shallow anterior chamber, corneal edema and elevated intraocular pressure despite maximum medical therapy was observed. Intraocular gas induced secondary pupillary block glaucoma diagnosis was made. She underwent intraocular lens reposition and peripheral iridectomy surgery in the same session. Intraocular pressure reduced dramatically after the procedure in addition to anterior chamber deepening. In this case report, we highlighted the very rare cause of pupillary block glaucoma and its management.
\end{abstract}

\section{Keywords}

Pupillary block glaucoma, Intraocular lens capture, Peripheral iridectomy, Intraocular gas injection

\section{Introduction}

Secondary pupillary block glaucoma is seen in $5-10 \%$ of all glaucoma types. Synechias between pupilla and crystalline/cataractous lens, intraocular lens (IOL) or vitreous may cause the deterioration of aqueous humor circulation and eventually lead to dramatic intraocular pressure elevation. Piggyback IOL, reverse implantation of angled IOL, silicon oil or gas bubbles in aphakics and intraocular tumors are among the rarer causes.

Pupillary capture occurs when part of the $I O L$ is displaced anteriorly in front of the pupil and it is typically noted as an early postoperative event, although a late form is also recognized [1]. It can be seen after scleral fixated IOL, in-the-bag IOL with large capsulorhexis, reverse implanted angled IOL and sulcus fixated IOL. This condition may not cause any symptom; however, anterior segment inflammation, visual distortion and IOP elevation due to pupillary block are not uncommon.

In the present study, we aimed to report the diagnosis and management of a patient with pupillary block glaucoma due to circumferential pupillary capture by an IOL secondary to intraocular gas injection.

\section{Case Report}

A 55-years-old female was admitted to our clinic with complaints of pain, redness and blurred vision in the right eye. She had a history of type II diabetes mellitus for 15 years. She had undergone a complicated cataract surgery with posterior capsule rupture and nucleus drop in the right eye two months ago in a foreign country. A few weeks later a pars plana vitrectomy, sulcus fixated threepiece intraocular lens implantation (IOL) and intraocular gas injection was performed in that eye. One week after this surgery, pain, redness and blurred vision in the right eye started. She was followed with a diagnosis of malignant glaucoma, hospitalized and treated medically. In spite of maximum medical treatment including systemic acetazolamide, the intraocular pressure (IOP) was over $30 \mathrm{mmHg}$ and pain was persistent. In our ophthalmic examination, best corrected visual acuity was counting fingers at 3 meters in the right eye and $20 / 40$ in the left eye. Slit-lamp biomicroscope showed a 360-degree pupillary capture by IOL, shallow anterior chamber and corneal edema (Figure 1A). IOP was 32 $\mathrm{mmHg}$ in the right eye and $16 \mathrm{mmHg}$ in the left eye. Left eye was also pseudophakic. Diabetic macular edema 


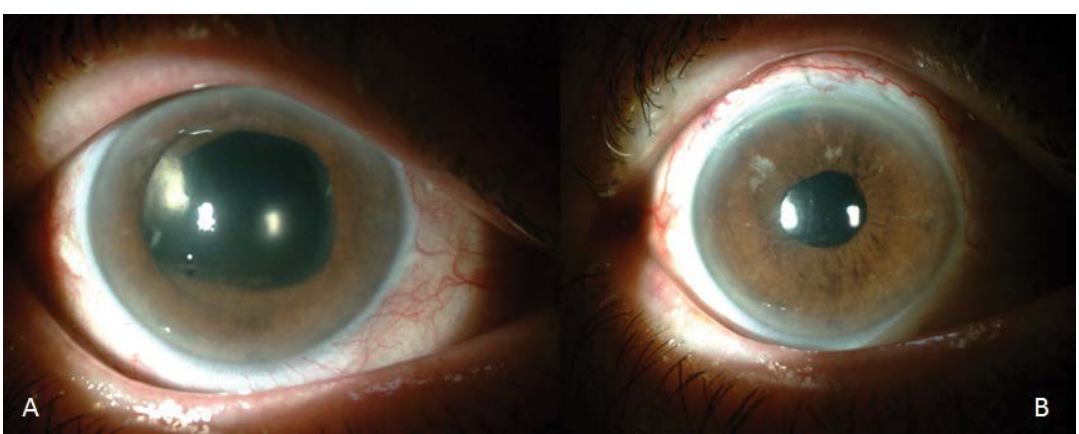

Figure 1: Circumferential Pupillary Capture by IOL.

A) Slit-lamp biomicroscope showed a 360-degree pupillary capture by IOL, shallow anterior chamber and corneal edema; B) After surgical correction of IOL malposition, patients' symptoms regressed dramatically.

was observed in the left eye. The details of posterior pole in the right eye could not be evaluated in detail. We thought as an angle closure glaucoma secondary to pupillary block due to forward displacement of IOL induced by intraocular gas. One session of maximum pupillary dilation and face down positioning was tried but no reversal was observed. Medical therapy including brimonidine tartrate $0.15 \%$ twice daily (Alphagan $\mathrm{P}$, Allergan, USA), dorzolamide hydrochloride $2 \%$ and timolol maleate $0.5 \%$ ophthalmic solution twice daily (Cosopt, Merck, USA), prednisolone acetate $1 \%$ every 6 hours (Pred Forte, Allergan, USA) $4 \times 1$ was started until surgery. An IOL reposition and peripheral iridectomy was performed successfully. After surgical correction of IOL malposition, patients' symptoms regressed dramatically (Figure 1B). Visual acuity was reached 20/40 and IOP was $14 \mathrm{mmHg}$ with a single antiglaucomatous agent in the right eye. The patient then referred to a retina specialist for further treatment of diabetic macular edema.

\section{Discussion}

Increased IOP due to intraocular surgery is relatively common and may be caused excessive inflammation, fibrin membrane, retained viscoelastic material, blood in the anterior chamber, intraocular gas, pupillary block glaucoma and secondary angle closure from anterior rotation of the ciliary body. It is important to determine the primary etiology of the elevated IOP to treat appropriately.

The injection of intraocular gas for retinal tamponade is not without risk for the anterior segment of the eye. Elevated intraocular pressure, migration of air bubbles, anterior displacement of the lens-iris diaphragm and refractive changes are the main disadvantages of the gas tamponades. In this case report, we have presented a patient with 360-degree pupillary capture by a threepiece sulcus fixated IOL most probably secondary to intraocular gas tamponade.

Postoperative pupillary block glaucoma has been reported following implantable collamer lens implantation, reverse implantation of single piece acrylic IOL, scleral fixated IOL implantation, air migration into the anterior chamber, air and silicone oil in phakics/ aphakics, vitreous prolapse, fibrin membrane formation, descemet stripping endothelial keratoplasty, iris fixated $\mathrm{IOL}$, and piggyback intraocular lens implantation [2-5]. In the present case, the pupillary block mechanism is presumed to be secondary to circumferential pupillary capture by IOL due to expansion of intraocular gas tamponade in the postoperative period. Increased intraocular pressure could be prevented by peripheral iridectomy (PI) in such case, however, during the second surgery PI did not performed. Therefore, we have performed IOL reposition and PI surgery for the resolution of pupillary block. After surgery, IOP returned to normal levels. However, the increase in visual acuity was not significant due to diabetic macular edema.

The main limitation of the present case report is the lack of evidence (ultrasound biomicroscope or anterior segment optical coherence tomography etc.) of intraocular gas during pupillary capture. This is related to misdiagnosed patient in an outside clinic and treated medically in the hospital about one week.

\section{Conclusion}

Although it is very rare condition intraocular tamponade gases may cause pupillary capture of IOL and pupillary block glaucoma. Differential diagnosis should be made in the light of patient history and clinical findings and treated promptly.

\section{References}

1. Ramos J, Jurado V (2011) Idiopathic pupillary capture 7 years after extracapsular cataract extraction and intraocular lens implantation. Digit J Ophthalmol 17: 36-37.

2. Al-Shahwan S, Ghadhfan F, Kalantan H, Al-Torbak AA (2006) Pupillary block glaucoma in phakic perfluoropropane gas filled eye. Br J Ophthalmol 90: 797-798.

3. Kim SK, Lanciano RC Jr, Sulewski ME (2007) Pupillary block glaucoma associated with a secondary piggyback intraocular lens. J Cataract Refract Surg 33: 1813-1814

4. Kumar A, Kedar S, Garodia VK, Singh RP (2002) Angle closure glaucoma following pupillary block in an aphakic perfluoropropane gas-filled eye. Indian J Ophthalmol 50: 220-221.

5. Li EY, Wu WK, Jhanji V (2011) Pupillary block glaucoma secondary to vitreous prolapse after Nd:YAG capsulotomy. Clin Exp Optom 94: 383-384. 